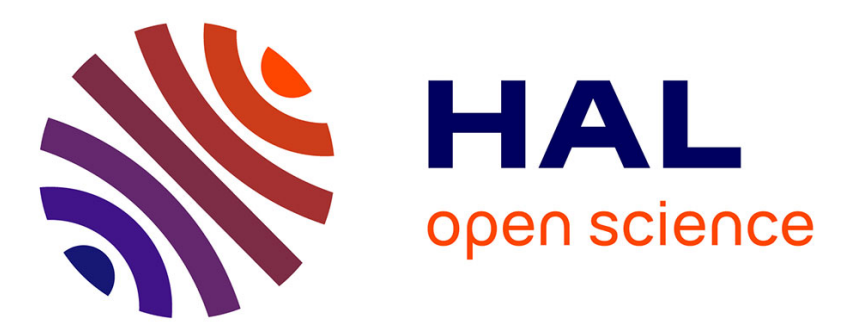

\title{
Event-triggered leader-following consensus of UAVs carrying a suspended load
}

\author{
A. Vega-Alonzo, J F Guerrero-Castellanos, Nicolas Marchand, S Durand, G. \\ Mino-Aguilar, V R Gonzalez-Díaz
}

\section{- To cite this version:}

A. Vega-Alonzo, J F Guerrero-Castellanos, Nicolas Marchand, S Durand, G. Mino-Aguilar, et al.. Event-triggered leader-following consensus of UAVs carrying a suspended load. EBCCSP 2019 - 5th IEEE Conference on Event-Based Control Communication and Signal Processing, May 2019, Vienne, Austria. 10.1109/EBCCSP.2019.8836720 . hal-02278014

\section{HAL Id: hal-02278014 https://hal.science/hal-02278014}

Submitted on 9 Dec 2019

HAL is a multi-disciplinary open access archive for the deposit and dissemination of scientific research documents, whether they are published or not. The documents may come from teaching and research institutions in France or abroad, or from public or private research centers.
L'archive ouverte pluridisciplinaire HAL, est destinée au dépôt et à la diffusion de documents scientifiques de niveau recherche, publiés ou non, émanant des établissements d'enseignement et de recherche français ou étrangers, des laboratoires publics ou privés. 
archives-ouvertes

\title{
Event-triggered leader-following consensus of UAVs carrying a suspended load
}

\author{
A. Vega-Alonzo, J Guerrero-Castellanos, Nicolas Marchand, S Durand, G. \\ Mino-Aguilar, V Gonzalez-Díaz
}

\section{To cite this version:}

A. Vega-Alonzo, J Guerrero-Castellanos, Nicolas Marchand, S Durand, G. Mino-Aguilar, et al.. Eventtriggered leader-following consensus of UAVs carrying a suspended load. 5th International Conference on Event-Based Control, Communication and Signal Processing (EBCCSP'19), 2019, Vienne, Austria. hal-02278014

\section{HAL Id: hal-02278014 \\ https://hal.archives-ouvertes.fr/hal-02278014}

Submitted on 9 Dec 2019

HAL is a multi-disciplinary open access archive for the deposit and dissemination of scientific research documents, whether they are published or not. The documents may come from teaching and research institutions in France or abroad, or from public or private research centers.
L'archive ouverte pluridisciplinaire HAL, est destinée au dépôt et à la diffusion de documents scientifiques de niveau recherche, publiés ou non, émanant des établissements d'enseignement et de recherche français ou étrangers, des laboratoires publics ou privés. 


\title{
Event-triggered leader-following consensus of UAVs carrying a suspended load
}

\author{
A. Vega-Alonzo*, J.F. Guerrero-Castellanos *, N. Marchand ${ }^{\dagger}$, S. Durand ${ }^{\ddagger}$, G. Mino-Aguilar* and V. R. Gonzalez-Díaz* \\ * Benemérita Universidad Autónoma de Puebla (BUAP), Facultad de Ciencias de la Electrónica, Puebla, Mexico \\ Email: fermi.guerrero@correo.buap.mx \\ ${ }^{\dagger}$ GIPSA-lab laboratory, Control Systems Dept., SySCo team \\ CNRS-Univ. of Grenoble, ENSE3 BP 46, 38402 St Martin d'Hères Cedex, France, \\ Email: nicolas.marchand@gipsa-lab.inpg.fr \\ ${ }^{\ddagger}$ ICube laboratory, INSA Strasbourg - Strasbourg univ. - CNRS, Strasbourg, France \\ Email: sylvain@durandchamontin.fr
}

\begin{abstract}
This article presents the design and development of an event-triggered control strategy to solve the problem of leader-following consensus and formation problem for a group of UAVs carrying a suspended load individually. In this work, the vehicles exchange information through a network, which is represented by a directed and strongly connected graph. Then, employing a decentralized control law, each UAV decides when it has to send a new value to its neighbors. The stability of the complete system is carried out, and numerical results show the advantages wrt information exchange between UAVs, as well as excellent performance in the angular stabilization and the minimum swing for the suspended load.
\end{abstract}

\section{INTRODUCTION}

Cooperative distributed control strategies for multiple vehicles have gained increased attention in recent years in the control community because such strategies provide attractive solutions to large-scale multi-agent problems, in which a group of coordinated agents is potentially able to outperform a single or several systems operating independently [1], [2], [3]. As an essential branch of cooperative control, distributed cooperative control of robotic vehicle networks has received much research attention [4], [5], in particular, a focus has been put to the problem of consensus and formation of under-actuated vertical take-off and landing (VTOL) unmanned aerial vehicles (UAVs) [6], [7], [8]. The main reason for this interest is the growing field of applications of VTOL-UAVs, such as individual transportation [9] or even collaborative transportation [10]. The development of transportation techniques with UAV represents many challenges, among which is the handling and control of the movements of the packages put due to their composition, weight or shape which can introduce disturbances and uncertainties leading instability [11].

The collaborative control approaches for VTOL-UAVs above

This work has been partially supported by CONACYT-ANUIES and ECOS-NORD under grant Mex. 296702 and Fra. M18M02. The second author acknowledges support from VIEP-BUAP, via the project number 100305333-VIEP2019. mentioned, consider vehicles with continuous-time dynamics, and it is assumed that each VTOL can broadcast its state and have continuous access to the states of their neighbors. However, in practical, continuous communication between vehicles is not possible, and it becomes essential to decide how frequently the aerial vehicles should communicate to preserve the closed-loop properties for the continuous-time design [12]. Furthermore, in Networked Control Systems (NCSs) and multi-agent scenarios where the control loop is closed over a communication link, energy consumption is correlated with the sampling rate of sensors, the rate at which processors recompute control inputs and actuator signals are transmitted. Then, performing these tasks periodically and at a high rate can be costly, might lead to inefficient implementations, or face hard physical constraints. To address these issues, the event-based paradigm, so-called event-triggered control [13], [14], [15], [16], [17], [18], appears as a mean to reduce the communication bandwidth in the network since, contrary to the classical scheme, an event-triggered can invoke a communication between the different agents only when a specific condition is satisfied, i.e., when an event is triggered. The event-triggered distributed control represents a trending topic and many groups around the world have carried out extensive research and reporting remarkable results, for instance, [19], [20], [21], [22], [23], [24]. Recently, in [25] an excellent review of event-triggered communication and control of networked systems for multi-agent consensus has appeared, where special attention was put to the assumptions on the capabilities of the network agents and the resulting features of the algorithm execution, including the interconnection topology, the evaluation of triggers, and the role of imperfect information. Although the event-triggered paradigm has shown advantages and benefits, there are only few reported works in the framework of aerial autonomous multi-vehicles where event-triggered feedback and event-triggered distributed control is developed and applied [26], [27].

In the presented paper a control strategy is developed for consensus and formation of a group of under-actuated planar vertical take-off and landing (PVTOL) unmanned aerial vehicles (UAVs) carrying a suspended load. 
The control strategy is two-layer based. Firstly, one designs an internal control law to stabilize the vertical and horizontal position and the angular dynamics originated by the suspended load. This inner layer allows taking into account the horizontal and pendulum dynamics such that minimum swing for the suspended load is achieved for each maneuver. The outer layer of the proposed strategy is charged with the event-triggered communication and control for the multi-vehicle system whose aim is to follow a virtual leader to achieve consensus and formation. The distributive event-triggered control proposed in the present paper is in the spirit of the ones proposed in [20], [21]. Whereas in [20] the agents' dynamics are represented by simple and double integrator, in [21] the agent's is represented by general linear dynamics. However, both approaches consider only the consensus problem and the communication between agents is represented by undirected graphs which is quite a restrictive condition i.e., only bidirectional communication is considered. Here the event-triggered distributed control technique is designed and applied to the problem of leader-following consensus and formation of a group of PVTOL-UAVs. The communication topology is represented by directed and connected graphs which represent a more realistic scenario. The practical convergence to leader is guaranteed under such an event-triggered strategy. Simulation is performed for the formation of four PVTOL-UAVs, where the effectiveness is illustrated and analyzed. Besides the development of a collaborative event-triggered control, the hoped-for contribution of this paper is that of unification, so that the reader can see how mixing topics such as Unmanned Aerial Vehicles and event-triggered collaborative control.

The rest of the paper is structured as follows. Section II contains mathematical preliminaries addressing the graph theory and the modeling of a PVTOL-UAV with a suspended load. In section III, the internal control is designed integrated by the attitude control, vertical control, horizontal and pendulum control for stabilizing a vertical and horizontal position and the angle of the suspended load of a PVTOL. In section IV, the event-triggered distributed control for a group of agents is developed. Section IV is devoted to simulation results, which show the effectiveness of the proposed algorithm. Finally, in section V the conclusions are presented.

\section{PRELIMINARIES}

\section{A. Graph theory}

Consider $\mathcal{G}=\{\mathcal{V}, \mathcal{E}\}$ consisting of a set of vertices (or nodes) $\mathcal{V}=1, \ldots, N$ and edges $\mathcal{E}$. If there is an edge $(i, j)$ between nodes $i$ and $j$, then $i$ and $j$ are called adjacent, i.e. $\mathcal{E}=(i, j) \in \mathcal{V} \times \mathcal{V}: i, j$ adjacent. $\mathcal{G}$ is called undirected if $(i, j) \in \mathcal{E} \Leftrightarrow(i, j) \in \mathcal{E}$. The adjacency matrix $A$ is defined by $a_{i j}=1$ if $i$ and $j$ are adjacent and $a_{i j}=0$ otherwise. A path from $i$ to $j$ is a sequence of distinct nodes, starting from $i$ and ending with $j$, such that each pair of consecutive nodes is adjacent. If there is a path from $i$ to $j$, then $i$ and $j$ are called connected. If all pairs of nodes in $\mathcal{G}$ are connected, then $\mathcal{G}$ is called connected. The distance $d(i, j)$ between two nodes is the number of edges of the shortest path from $i$ to $j$. The diameter $d$ of $\mathcal{G}$ is the maximum distance $d(i, j)$ over all pairs of nodes. The degree matrix $D$ of $\mathcal{G}$ is the diagonal matrix with elements $d_{i}$ equal to the cardinality of node i's neighbor set $\mathcal{N}_{i}=\{j \in \mathcal{V}:(i, j) \in \mathcal{E}\}$. The Laplacian matrix $\mathcal{L}$ of $\mathcal{G}$ is defined as $\mathcal{L}=D-A$. For undirected graphs, $\mathcal{L}$ is symmetric and positive semi-definite, i.e., $\mathcal{L}=\mathcal{L}^{T} \geq 0$. The row sums of $\mathcal{L}$ are zero. Thus, the vector of ones is an eigenvector corresponding to eigenvalue $\lambda_{i}(\mathcal{G})=0$, i.e., $\mathcal{L} 1=0$. For connected graphs, $\mathcal{L}$ has exactly one zero eigenvalue, and the eigenvalues can be listed in increasing order $0=\lambda_{1}(\mathcal{G})<\lambda_{2}(\mathcal{G}) \leq \ldots \leq \lambda_{N}(\mathcal{G})$. The second eigenvalue $\lambda_{2}(\mathcal{G})$ is called the algebraic connectivity. A component of graph $\mathcal{G}$ is a connected subgraph that is maximal. The leader is represented by vertex 0 and information is exchanged between the leader and the agents which are in the neighbors of the leader. Then, we have a graph $\overline{\mathcal{G}}$, which consists of graph $\mathcal{G}$, vertex 0 and edges between the leader 0 and its neighbors. Furthermore, let $G=\operatorname{diag}\left(g_{1}, \ldots, g_{N}\right)$ be the diagonal matrix of pinning gains which describes the connections between the leader and the follower nodes [28], [3].

Lemma 2.1: The matrix $H=\mathcal{L}+G$ corresponding to a graph $\overline{\mathcal{G}}$ has the following properties:

1) The matrix $H$ has nonnegative eigenvalues;

2) The matrix $H$ is positive definite if and only if the graph $\overline{\mathcal{G}}$ is connected.

\section{B. Modeling}

The modeling of this system has been studied in the literature, and the mathematical model is now well known (e.g. [9]). However, for clearness, this will be briefly presented here. Consider the UAV carrying a suspended mass, as shown in Fig. 1. Let $p_{M}=(x z)^{T} \in \mathbb{R}^{2}$ describe the position of the center of mass of the UAV in the inertial reference frame $\mathbf{E}^{f}$ and $\theta \in \mathbb{R}$ be the angle between the horizon and the UAV. Moreover, let $\alpha \in \mathbb{R}$ be the rotation angle between the gravity vector and the cable of length $L$ connecting $p_{M}$ to the suspended mass. The vehicle is actuated by two propellers generating the only positive forces $f_{1}, f_{2} \in \mathbb{R}$. To simplify the notation, the system inputs are defined as $u_{1}=f_{1}+f_{2}$ and $u_{2}=\left(f_{1}-f_{2}\right) l$ where $l$ is the distance between the motors and the center of mass. Note that $u_{1}$ and $u_{2}$, represent the total thrust and the moment generated by the propellers, respectively. Assuming that the cable is rigid, inextensible and massless, the following dynamical model is obtained [9]:

$$
\begin{array}{r}
(M+m)(\ddot{z}+g)+m L\left(\cos \alpha \dot{\alpha}^{2}+\sin \alpha \ddot{\alpha}\right)=u_{1} \cos \theta \\
(M+m) \ddot{x}+m L\left(\sin \alpha \dot{\alpha}^{2}-\cos \alpha \ddot{\alpha}\right)=u_{1} \sin \theta \\
m L^{2} \ddot{\alpha}+m L \sin \alpha \ddot{z}-m L \cos \alpha \ddot{x}+m g L \sin \alpha=0 \\
J \ddot{\theta}=u_{2}
\end{array}
$$

where $M$ and $m$ are the mass of the UAV and the load respectively, $g$ is the gravitational constant, $J$ is the inertia of the UAV.

System (1) can be decoupled and the following dynamic model is obtained 


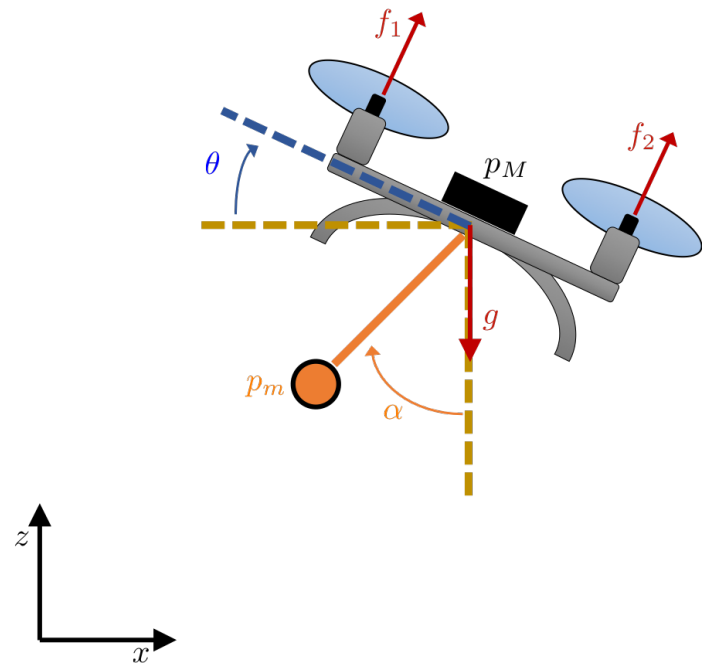

Fig. 1. PVTOL

$$
\Sigma_{T}:=\left\{\begin{array}{r}
\ddot{z}=f_{z}(\alpha, \theta)+g_{z}(\alpha, \theta) u_{1} \\
\ddot{x}=f_{x}(\alpha, \theta)+g_{x}(\alpha, \theta) u_{1} \\
\ddot{\alpha}=g_{\alpha}\left(g_{\alpha}, \theta\right) u_{1}
\end{array}\right.
$$

and

$$
\Sigma_{R}:=\left\{\ddot{\theta}=J^{-1} u_{2}\right.
$$

where

$$
\begin{array}{r}
f_{z}(\alpha, \theta)=-g-\frac{m}{M+m} L \cos \alpha \dot{\alpha}^{2} \\
f_{x}(\alpha, \theta)=-\frac{m}{M+m} L \sin \alpha \dot{\alpha}^{2} \\
g_{z}(\alpha, \theta)=\frac{1}{M+m}\left(\cos \theta+\frac{m}{2 M}(\cos \theta-\cos (\theta-2 \alpha))\right) \\
g_{x}(\alpha, \theta)=\frac{1}{M+m}\left(\sin \theta+\frac{m}{2 M}(\sin \theta-\sin (\theta-2 \alpha))\right) \\
g_{\alpha}(\alpha, \theta)=\frac{1}{M L} \sin (\theta-\alpha)
\end{array}
$$

Note that (2) and (3) constitute a cascade system. $\Sigma_{T}$ represents the vertical-horizontal and pendulum dynamics which depends on $\Sigma_{R}$, whereas $\Sigma_{R}$ represents the attitude which does not depend on $\Sigma_{T}$. As a consequence $u_{2}$ can be firstly and independently designed.

\section{INNER CONTROL DESIGN}

In this section, we are interested in the design of an internal control to stabilize the vertical and horizontal position and the angular dynamics originated by the suspended load which can be viewed as a pendulum.

\section{A. Attitude control}

Firstly, one proposes a simple and robust control law that stabilizes the attitude.

Definition 3.1: Given a positive constant $M$, a continuous, non-decreasing function $\sigma_{M}: \mathbb{R} \rightarrow \mathbb{R}$ is defined by

(1) $\sigma_{M}(s)=s$ if $|s|<M$;

(2) $\sigma_{M}(s)=\operatorname{sign}(s) M$ elsewhere
Then, one has the following result inspired in this one reported in [29] for the attitude stabilization of rigid bodies.

Proposition 3.2: Consider the attitude dynamics described by (3) with the following bounded control input

$$
u_{2}=-\sigma_{M_{2}}\left(a_{1} \dot{\theta}+\sigma_{M_{1}}\left(a_{2} \dot{\theta}+a_{1} a_{2}\left(\theta-\theta_{D}\right)\right)\right)
$$

where $\sigma_{M}$. is defined in (9) with $M_{2}>2 M_{2} . a_{1}, a_{2} \in \mathbb{R}_{+}$are tuning parameters. Then the input (10) stabilizes global and asymptotically the UAV's attitude to the desired attitude $\theta_{D}$.

Proof: The proof follows the ones presented in [29], [30].

\section{B. Vertical control}

Now consider the vertical dynamics described by $\ddot{z}=$ $f_{z}(\alpha, \theta)+g_{z}(\alpha, \theta) u_{1}$ with the following control law

$$
u_{1}=\frac{1}{g_{z}(\alpha, \theta)}\left(-f_{z}(\alpha, \theta)+r_{z}\right)
$$

consequently, the closed-loop the vertical dynamics becomes

$$
\ddot{z}=r_{z}
$$

with $r_{z}$ to be defined later.

\section{Horizontal and pendulum control}

Consider the horizontal and pendulum dynamics

$$
\Sigma_{H P}:=\left\{\begin{array}{r}
\ddot{x}=f_{x}(\alpha, \theta)+g_{x}(\alpha, \theta) u_{1} \\
\ddot{\alpha}=g_{\alpha}\left(g_{\alpha}, \theta\right) u_{1}
\end{array}\right.
$$

Assume that using the control law (10), one can stabilize the attitude dynamics of PVTOL, that is $\theta=\theta_{D} \approx 0$. Then, using the trigonometric identities: $\cos \left(\theta_{D}-2 \alpha\right)=\cos 2 \alpha+\theta_{D} \sin 2 \alpha$ and $\sin \left(\theta_{D}-2 \alpha\right)=\theta_{D} \cos 2 \alpha-\sin 2 \alpha$ and after a sufficiently long time, system (12) becomes

$$
\begin{aligned}
& \ddot{x}=f_{1}\left(\alpha, u_{1}\right)+g_{1}\left(\alpha, u_{1}\right) \theta_{D} \\
& \ddot{\alpha}=f_{2}\left(\alpha, u_{1}\right)+g_{2}\left(\alpha, u_{1}\right) \theta_{D}
\end{aligned}
$$

where

$$
\begin{aligned}
f_{1}\left(\alpha, u_{1}\right) & =-\frac{1}{M+m}\left(m L \sin \alpha \dot{\alpha}^{2}+u_{1} \sin \alpha\right) \\
g_{1}\left(\alpha, u_{1}\right) & =-\frac{u_{1}}{M+m}\left(1+\frac{m}{2 M}(1+\cos (2 \alpha))\right) \\
f_{2}\left(\alpha, u_{1}\right) & =-\frac{u_{1}}{M L} \sin \alpha \\
g_{2}\left(\alpha, u_{1}\right) & =-\frac{u_{1}}{M L} \cos \alpha
\end{aligned}
$$

Let's define $F=x+\alpha$, then one has the following

$$
\ddot{F}=\bar{f}\left(\alpha, u_{1}\right)+\bar{g}\left(\alpha, u_{1}\right) \theta_{D}
$$

with

$$
\begin{aligned}
& \bar{f}\left(\alpha, u_{1}\right)=f_{1}\left(\alpha, u_{1}\right)+f_{2}\left(\alpha, u_{1}\right) \\
& \bar{g}\left(\alpha, u_{1}\right)=g_{1}\left(\alpha, u_{1}\right)+g_{2}\left(\alpha, u_{1}\right)
\end{aligned}
$$

Note that $\theta_{D}$ becomes an input, then choosing

$$
\theta_{D}=\frac{1}{\bar{g}\left(\alpha, u_{1}\right)}\left(-\bar{f}\left(\alpha, u_{1}\right)+r_{F}\right)
$$


the closed-loop system for the horizontal and pendulum dynamics becomes

$$
\ddot{F}=r_{F}
$$

Consequently, from (11) and (17) the closed-loop overall system (2) can be written as follows

$$
\Sigma_{P V T O L}:=\left\{\begin{array}{l}
\ddot{z}=r_{z} \\
\ddot{F}=r_{F}
\end{array}\right.
$$

Note that $r_{z}$ and $r_{F}$ can be chosen for regulation or tracking purposes. Furthermore, the selection of $F=x+\alpha$ allows taking into account the horizontal and pendulum dynamics such that minimum swing for the suspended load is achieved for each maneuver. The design of $r_{z}$ and $r_{F}$ will be carried out in the next section in a framework of cooperative control.

\section{EVENT-TRIGGERED COOPERATIVE CONTROL}

In this section, we are interested in the design of a control law to obtain consensus and formation of multiples PVTOL-UAVs carrying a suspended load, as represented in Figure 2. For that, we consider a group of vehicles described by equation (1). Besides, we assume that control signals $u_{1}$ and $u_{2}$ are applied for an inner control-loop. Then, for leader-following consensus control purposes, each follower vehicle is modeled by equation (18), that is

$$
\Sigma_{P V T O L_{i}}:=\left\{\begin{array}{l}
\ddot{z}_{i}=r_{z_{i}} \\
\ddot{F}_{i}=r_{F_{i}}
\end{array}\right.
$$

with $i \in \mathcal{V}$. Let's define the following variables: $\xi_{i_{1}}=z_{i}$, $\xi_{i_{2}}=\dot{z}_{i}, \xi_{i_{3}}=F_{i}, \xi_{i_{4}}=\dot{F}_{i}$. Then, system (19) can be written as

$$
\dot{\xi}_{i}=\bar{A} \xi_{i}+\bar{B} \bar{u}_{i}
$$

where $\xi_{i}=\left(\xi_{1_{i}} \xi_{2_{i}} \xi_{3_{i}} \xi_{4_{i}}\right)^{T}, \bar{u}_{i}=\left(r_{z_{i}} r_{F_{i}}\right)^{T}, \bar{A} \in \mathbb{R}^{4 \times 4}$, $\bar{B} \in \mathbb{R}^{4 \times 2}$. Furthermore, the dynamics of a leader, labeled 0 is given by

$$
\dot{\xi_{0}}=\bar{A} \xi_{0}
$$

where $\xi_{0} \in \mathbb{R}^{4}$. The leader vehicle can be considered as a command generator exosystem that generates the desired target reference or trajectory. The objective is to design local controllers $\bar{u}_{i}$ for all follower nodes.
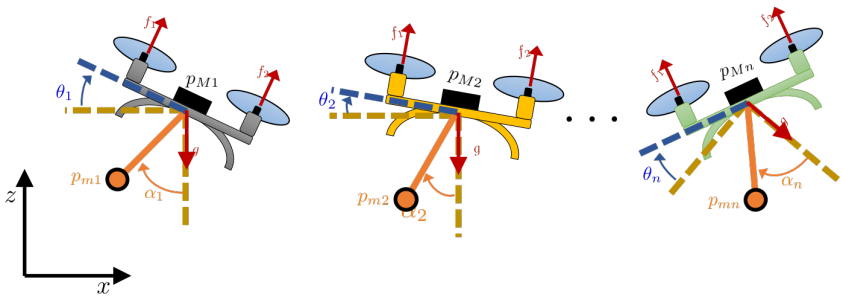

Fig. 2. Group of $N$-PVTOL

Definition 4.1: The event-triggered leader-following consensus of system (20)-(21) is said to be practically achieved if, for each vehicle $i \in \mathcal{V}$, there is a local state feedback $\bar{u}_{i}$ which depends on $m_{i}$ and $m_{j}$, with $j \in \mathcal{N}_{i}$ (node $i$ 's neighbor set), such that the closed-loop system satisfies

$$
\lim _{t \rightarrow \infty}\left\|\xi_{i}(t)-\xi_{0}(t)\right\|=\Delta, \quad i=1, \ldots, N
$$

for any initial condition $\xi_{i}(0), i=0,1, \ldots, N$. Note that $m_{i}$ is the latest broadcast state of vehicle $i$, i.e. $m_{i}(t)=\xi_{i}\left(t_{k}^{i}\right), t \in$ $\left[t_{k}^{i}, t_{k+1}^{i}\left[\right.\right.$, where $t_{0}^{i}, t_{1}^{i} \ldots$ is the sequence of event times of vehicle $i$.

Then, we have our main result.

Theorem 4.2: Consider the multi-vehicle system (20)-(21), under the following control law

$$
\bar{u}_{i}=K\left[\sum_{j \in \mathcal{N}_{i}}\left(m_{j}-m_{i}\right)+g_{i}\left(\xi_{0}-m_{i}\right)\right]
$$

with $K=\rho \bar{B}^{T} P$, being $P$ a solution to the Riccati equation

$$
\bar{A}^{T} P+P \bar{A}-2 \rho P \bar{B} \bar{B}^{T} P=-Q
$$

Suppose the trigger function is given by:

$$
e_{i}\left(\xi_{i}, m_{i}\right)=\tilde{e}_{i_{1}} \wedge \tilde{e}_{i_{2}} \wedge \tilde{e}_{i_{3}} \wedge \tilde{e}_{i_{4}}
$$

where $e_{i_{s}}=\left|\bar{e}_{i_{s}}\right|-\delta$ with $\bar{e}_{i_{s}}=m_{i_{s}}-\xi_{i_{s}}$, and $\tilde{e}_{i_{s}}=1$ if $e_{i_{s}} \geq 0$ and $\tilde{e}_{i_{s}}=0$ elsewhere. $\delta \in \mathbb{R}_{+}, i \in \mathcal{V}$ and $s=$ $\{1,2,3,4\}$. Then, all the agents follow the leader from any initial condition and they converge to a neighborhood given by

$$
\lim _{t \rightarrow \infty}\|\varepsilon(t)\|=\frac{2 \sqrt{N} \delta\|\tilde{B}\| \bar{\alpha}}{\lambda_{1}^{\tilde{A}}}=\Delta
$$

where $\underset{\sim}{\varepsilon}=\left(\varepsilon_{1}, \varepsilon_{2}, \ldots, \varepsilon_{N}\right)^{T}$ and $\varepsilon_{i}=\xi_{i}-\xi_{0}$. Moreover, $\lambda_{1}^{\tilde{A}}=$ $\lambda_{\min }(\tilde{A})$ being $\tilde{A}=I_{N} \otimes \bar{A}-\tilde{B}, \bar{\alpha}=\|R\|\left\|R^{T}\right\|$, with $R$ a matrix which diagonalizes matrix $\tilde{A}$. $\tilde{B}=-H \otimes \bar{B} K$, with $H=\mathcal{L}+G$.

Proof: Let's define the following error variables $\bar{e}_{i}=$ $m_{i}-\xi_{i}$ and $\varepsilon_{i}=\xi_{i}-\xi_{0}$, then, the control law (22) can be written as follows

$$
\bar{u}_{i}=K\left[\sum_{j \in \mathcal{N}_{i}}\left(\varepsilon_{j}-\varepsilon_{i}\right)+g_{i} \varepsilon_{i}+\sum_{j \in \mathcal{N}_{i}}\left(\bar{e}_{j}-\bar{e}_{i}\right)-g_{i} \bar{e}_{i}\right]
$$

and the closed-loop system becomes

$$
\begin{aligned}
\dot{\varepsilon}_{i}= & \bar{A} \varepsilon_{i}+ \\
& \bar{B}\left(\begin{array}{c}
K \sum_{j \in \mathcal{N}_{i}}\left(\varepsilon_{j}-\varepsilon_{i}\right)+K g_{i} \varepsilon_{i} \\
+K \sum_{j \in \mathcal{N}_{i}}\left(\bar{e}_{j}-\bar{e}_{i}\right)-K g_{i} \bar{e}_{i}
\end{array}\right) \\
& \begin{array}{c}
+\bar{A} \varepsilon_{i} \\
+
\end{array} \\
& +\bar{B} K \sum_{j \in \mathcal{N}_{i}}\left(\varepsilon_{j}-\varepsilon_{i}\right)+\bar{B} K g_{i} \varepsilon_{i} \\
& +\bar{B} K \sum_{j \in \mathcal{N}_{i}}\left(\bar{e}_{j}-\bar{e}_{i}\right)-\bar{B} K g_{i} \bar{e}_{i}
\end{aligned}
$$

By introducing $\varepsilon=\left(\varepsilon_{1}, \varepsilon_{2}, \ldots, \varepsilon_{N}\right)^{T}, \quad G=$ $\operatorname{diag}\left(g_{1}, g_{2}, \ldots, g_{N}\right), \bar{e}=\left(\bar{e}_{1}, \bar{e}_{2}, \ldots, \bar{e}_{N}\right)^{T}$ and by using the Laplaciane $\mathcal{L}$ of the graph $\mathcal{G}$, one has

$$
\dot{\varepsilon}=[\underbrace{(\underbrace{}_{N} \otimes \bar{A})-H \otimes \bar{B} K}_{\tilde{A}}] \varepsilon+[\underbrace{-H \otimes \bar{B} K}_{\tilde{B}}] \bar{e}
$$

where $H=\mathcal{L}+G$. Lemma 2.1 states the properties of $H$. To continue with the proof, firstly, one assumes $\bar{e}=0$, and it 
will be shown $\dot{\varepsilon}=\tilde{A} \varepsilon$ is asymptotically stable. For that, let's consider the following Lyapunov function

$$
V(\varepsilon)=\varepsilon^{T}\left(I_{N} \otimes P\right) \varepsilon
$$

whose derivative along the trajectories of (24) is

$$
\begin{aligned}
\dot{V}(\varepsilon) & =\varepsilon^{T}\left[\left(I_{N} \otimes \bar{A}^{T}\right)-\left(H \otimes K^{T} \bar{B}^{T}\right)\left(I_{N} \otimes P\right)\right] \varepsilon \\
& +\varepsilon^{T}\left[\left(I_{N} \otimes P\right)\left(I_{N} \otimes \bar{A}-H \otimes \bar{B} K\right)\right] \varepsilon \\
& =\varepsilon^{T}\left[I_{N} \otimes\left(P \bar{A}+\bar{A}^{T} P\right)-H \otimes\left(2 P \bar{B} \bar{B}^{T} P\right)\right] \varepsilon
\end{aligned}
$$

Since $H$ is symmetric, there exists a matrix $T \in \mathbb{R}^{N \times N}$ such that $T H T^{T}=\Lambda:=\operatorname{diag}\left(\lambda_{1}, \ldots, \lambda_{N}\right)$ where $\lambda_{1}, \ldots, \lambda_{N}$ are the eigenvalues of $H$ which are positive (see Lemma 2.1). has

Let $\tilde{\varepsilon}=\left(T \otimes I_{N}\right) \varepsilon$ be a linear transformation, then one

$$
\begin{aligned}
\dot{V} & =\tilde{\varepsilon}^{T}\left[\left(I_{N} \otimes\left(P \bar{A}+\bar{A}^{T} P\right)\right)-\Lambda \otimes\left(2 P \bar{B} \bar{B}^{T} P\right)\right] \tilde{\varepsilon} \\
& \leq \sum_{i=1}^{N} \tilde{\varepsilon}_{i}^{T}\left[P \bar{A}+\bar{A}^{T} P-\lambda_{i}\left(2 P \bar{B} \bar{B}^{T} P\right)\right] \tilde{\varepsilon}_{i} \\
& \leq-\sum_{i=1}^{N} \tilde{\varepsilon}_{i}^{T} Q \tilde{\varepsilon}_{i} \\
& \leq-\sum_{i=1}^{N} \varepsilon_{i}^{T} \underbrace{\left(I_{N} \otimes T^{T}\right) Q\left(T \otimes I_{N}\right)}_{\bar{Q}} \varepsilon_{i}<0 \quad \forall \quad \varepsilon_{i} \neq 0
\end{aligned}
$$

$\Longrightarrow \bar{A}$ is Hurwitz and $\varepsilon_{i} \rightarrow 0$ when $\bar{e}=0$ and $t \rightarrow \infty$.

Now, the case $\bar{e} \neq 0$ is considered and assuming that $\lambda_{1}^{\tilde{A}}=$ $\lambda_{\min }(\tilde{A})$, then the solution of

$$
\dot{\varepsilon}=\tilde{A} \varepsilon+\tilde{B} \bar{e}
$$

is given by

$$
\begin{aligned}
\varepsilon(t) & =e^{\tilde{A} t} \varepsilon(0)+\int_{0}^{t} e^{\tilde{A}(t-\tau)} \tilde{B} \bar{e}(\tau) d \tau \\
\|\varepsilon(t)\| & \leq\left\|e^{\tilde{A} t} \varepsilon(0)\right\|+\int_{0}^{t}\left\|e^{\tilde{A}(t-\tau)} \tilde{B} \bar{e}(\tau) d \tau\right\|
\end{aligned}
$$

Furthermore, let $R \tilde{A} R^{T}=\Phi=\operatorname{diag}\left(\lambda_{1}^{\tilde{A}}, \ldots, \lambda_{N}^{\tilde{A}}\right)$ be a diagonal matrix, it results

$$
\|\varepsilon(t)\| \leq \bar{\alpha} e^{-\lambda_{1}^{\tilde{A}} t}\|\varepsilon(0)\|+\bar{\alpha} \int_{0}^{t} e^{-\lambda_{1}^{\tilde{A}}(t-\tau)}\|\tilde{B} \bar{e}(\tau)\| d \tau
$$

where

$$
\bar{\alpha}=\|R\|\left\|R^{T}\right\|
$$

since $\|\tilde{B} \bar{e}\| \leq\|\tilde{B}\|\|\bar{e}\|$, and due to the event condition, one has

$$
\|\bar{e}\|=\sqrt{4 \delta^{2}+4 \delta^{2}+\ldots+4 \delta^{2}}=2 \delta \sqrt{N}
$$

Substituting, the error is bounded as follows

$$
\begin{aligned}
\|\varepsilon(t)\| \leq & \bar{\alpha} e^{-\lambda_{1}^{\tilde{A}} t}\|\varepsilon(0)\|+\bar{\alpha} \int_{0}^{t} e^{-\lambda_{1}^{\tilde{A}}(t-\tau)} 2 \delta \sqrt{N}\|\tilde{B}\| d \tau \\
\leq & \bar{\alpha} e^{-\lambda_{1}^{\tilde{A}} t}\|\varepsilon(0)\|+2 \bar{\alpha} e^{-\lambda_{1}^{\tilde{A}} t}\|\tilde{B}\| \delta \sqrt{N} \int_{0}^{t} e^{\lambda_{1}^{\tilde{A}} \tau} d \tau \\
\leq & \bar{\alpha} e^{-\lambda_{1}^{\tilde{A}} t}\|\varepsilon(0)\| \\
& +2 \bar{\alpha} e^{-\lambda_{1}^{\tilde{A}} t}\|\tilde{B}\| \delta \sqrt{N}\left(\frac{e^{\lambda_{1}^{\tilde{A}} t}}{\lambda_{1}^{\tilde{A}}}-\frac{1}{\lambda_{1}^{\tilde{A}}}\right)
\end{aligned}
$$

$$
\|\varepsilon(t)\| \leq \frac{2 \bar{\alpha} \delta \sqrt{N}\|\tilde{B}\|}{\lambda_{1}^{\tilde{A}}}=\Delta
$$

As a consequence, with the event-triggered distributive control law, the leader-following consensus is achieved practically, i.e. the error between the followers and the leader converges to a ball centered at the origin with radius $\Delta$.

\section{A. Formation control}

Formation control is characterized as geometrical patterns to be realized by the vehicle group. The event-triggered leader-following consensus control law (22) can be extended to leader-following formation. In this case, followers should move along a leader's trajectory or target reference while maintaining the desired shape.

Let $\Upsilon$ be a set of relative, desired inter-agent distances, that is,

$$
\Upsilon=\left\{\varrho_{i j} \in \mathbb{R} \mid \varrho_{i j}>0, i, j=1, \ldots, N, i \neq 0\right\}
$$

with $\varrho_{i j}=\varrho_{j i}$ and where it is assumed that $\Upsilon$ is a feasible formation, that is, there are points $\zeta_{1}, . ., \zeta_{N} \in \mathbb{R}^{4}$ such that

$$
\left\|\zeta_{i}-\zeta_{j}\right\|=\varrho_{i j}
$$

Then, consensus algorithm is extended to formation control if the formation is represented by vectors of relative positions of neighboring agents. In this case, the control law (22) becomes

$$
\bar{u}_{i}=K\left[\sum_{j \in \mathcal{N}_{i}}\left(m_{j}-m_{i}\right)-\left(\zeta_{j}-\zeta_{i}\right)+g_{i}\left(\xi_{0}-m_{i}\right)\right]
$$

Note that since one desires that followers achieve a target reference with zero velocity (similar to regulation case) then, second and fourth component of vectors $\zeta_{i}$ and $\zeta_{j}$ must to be zero, i.e., $\zeta_{i_{2}}=\zeta_{i_{4}}=\zeta_{j_{2}}=\zeta_{j_{4}}=0$ for all $i, j=1, \ldots, N$.

\section{Simulation Results}

In order to test and validate the efficiency of the proposed control law, a set of numerical simulations were performed. For the numerical simulations, one considers four agents, where a PVTOL represents each agent. Both the mathematical model of the PVTOL and the proposed event-triggered cooperative control law were simulated in the MATLAB/Simulink software. During the simulations, an interconnection between the agents was considered as shown in Figure 3, where only agent 1 (PVTOL 1) receives information from the leader. Note that Figure 3 represents a direct and connect graph.

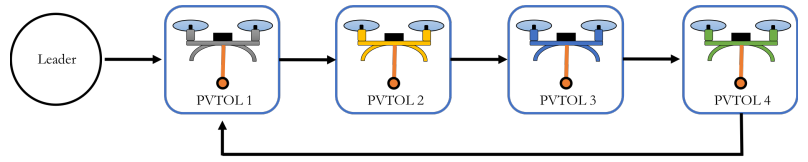

Fig. 3. Graph of interconnection between the PVTOLs

The simulations aimed to verify that the developed control law drives each agent to the desired formation, maintaining the consensus between them while removing the oscillation created by the suspended load of each agent. Note that the leader vehicle is a command generator which generates 
the desired target reference. For the following numerical simulation, the target reference is given by $\xi_{0}=(0,0,4,0)$. Then, followers must achieve consensus in this position. However, using control law (28) one obtains a leader-following formation. Note that, the desired formation refers to the positions that each agent must reach and maintain after the consensus with respect to the leader is achieved. The desired formation for the presented simulations is shown in Table I.

\begin{tabular}{|c|c|}
\hline Agent & $\left(\zeta_{i_{1}}, \zeta_{i_{2}}, \zeta_{i_{3}}, \zeta_{i_{4}}\right)$ \\
\hline PVTOL 1 & $(5,0,0,0)$ \\
\hline PVTOL 2 & $(2,0,0,0)$ \\
\hline PVTOL 3 & $(-1,0,0,0)$ \\
\hline PVTOL 4 & $(-4,0,0,0)$ \\
\hline
\end{tabular}

TABLE I. DESIRED FORMATION FOR AGENTS.

For the simulation, different initial conditions were established for the positions of the agents and the angle generated by the suspended load of each agent. The initial conditions' numerical values for each agent are depicted in table II.

\begin{tabular}{|c|c|c|}
\hline Agent & $\xi_{i}(0)=\left(\xi_{1_{i}}(0), \xi_{2_{i}}(0), \xi_{3_{i}}(0), \xi_{4_{i}}(0)\right)$ & Load angle $(\alpha(0))$ \\
\hline PVTOL 1 & $(3,0,0,0)$ & $28.6^{\circ}$ \\
\hline PVTOL 2 & $(1,0,0,0)$ & $-28.6^{\circ}$ \\
\hline PVTOL 3 & $(-1,0,0,0)$ & $17.2^{\circ}$ \\
\hline PVTOL 4 & $(-2,0,0,0)$ & $0^{\circ}$ \\
\hline
\end{tabular}

TABLE II. INITIAL CONDITIONS FOR AGENTS

The obtained results are depicted in the following. The agents' state evolution is shown in Figure 4-6. During the simulation, disturbances forces are introduced to show the efficiency of the proposed control strategy. In the second 40, a disturbance affects the suspended load of PVTOL-3. The second disturbance occurs in the second 55, where the position of PVTOL-2 is altered, changing both the $x$ and $z$ position of the PVTOLs' group.

Figure 4 shows the evolution of the position of the agents on the $x$-axis. It is observed that each PVTOL begins in its initial conditions and approximately in the second 30 reaches the desired formation and maintains a constant position until the first disturbance in the second 40. PVTOL 4 shows more significant movement, while the other agents also have slight changes. Later in the second 55, the second perturbation occurs, the evolution of the positions on the $x$-axis shows how the control algorithm leads the agents to their desired location in spite of the disturbance.

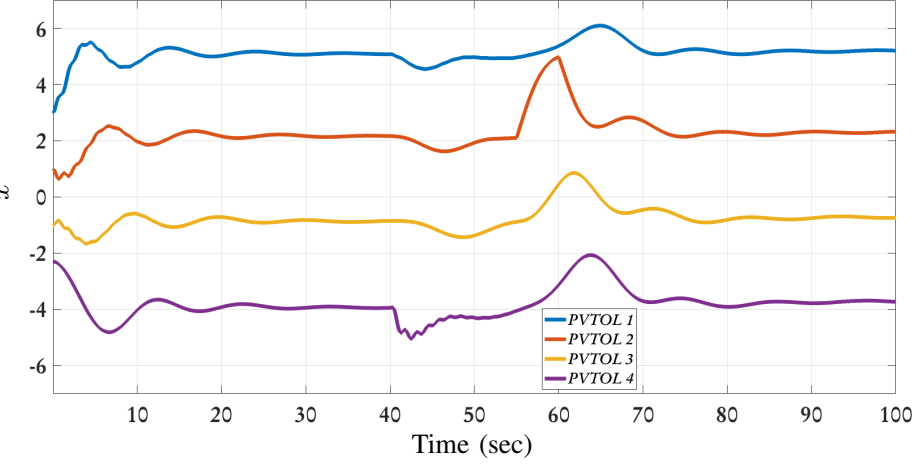

Fig. 4. Evolution along the $x$ axis

The evolution of the position of the PVTOLs on the $z$-axis is shown in Figure 5. For the present work, the $z$-axis corresponds to the height, so all agents begin at 0 . It is observed that the PVTOLs have a movement on the $z$-axis until they reach the desired formation. The first perturbation is applied to the suspended load of the PVTOL 4, but it can be seen that it does not affect the z-axis. However, the second disturbance generates a change in the positions of the PVTOLs. After a sufficiently long time, the desired formation is reached.

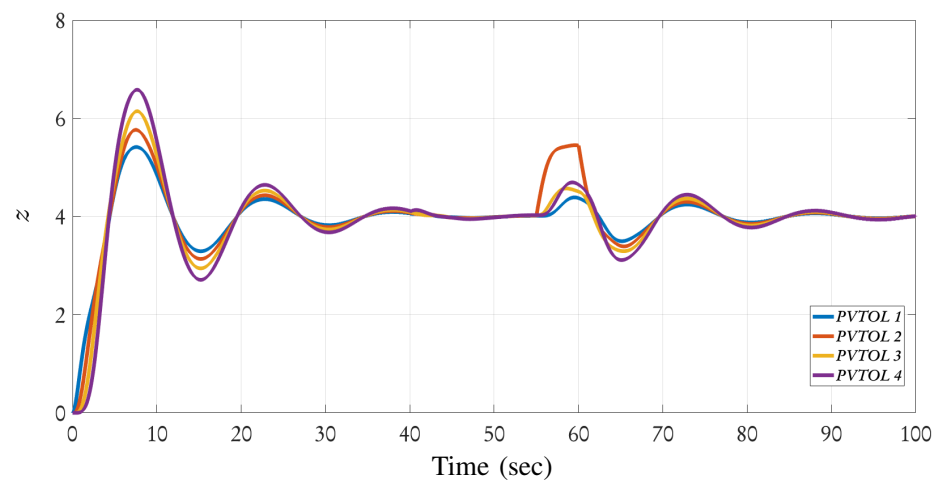

Fig. 5. Evolution along the $z$ axis

Figure 6 shows the angles' evolution of each agent's load (depicted in degrees). The angles' load initial conditions were defined with non-zero values for the different agents. Note that approximately in the second 20, the oscillations of the suspended loads have been removed. Then, the first disturbance is applied to the second 40, causing an abrupt change of the angle $\alpha$ of the PVTOL 4. The control law reduces the oscillations caused by the disturbance. Also, the changes produced by the second disturbance are removed approximately for the second 80 . 


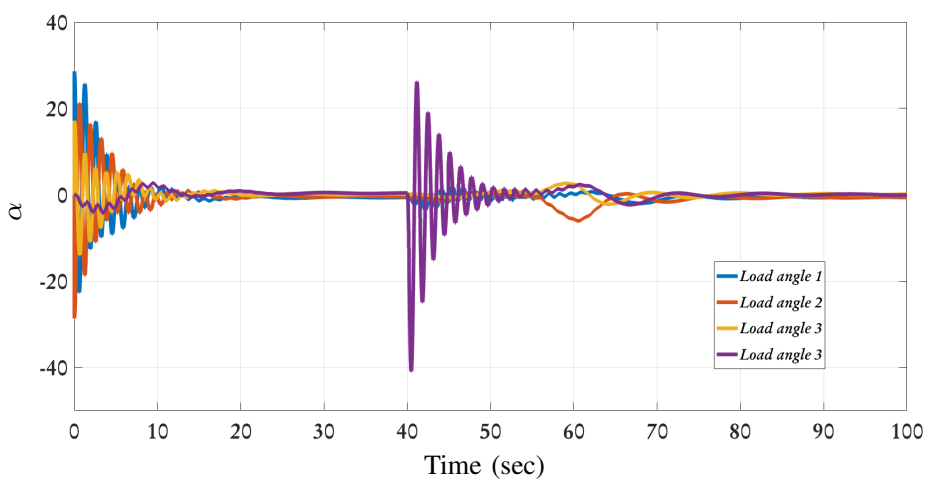

Fig. 6. Evolution of each load angle

Note that when an event is generated, the state of an agent is transmitted to its neighbors. Figure 7 shows the evolution of the number of events generated by each of the agents during the simulation. Also, the counting of transmissions that would be issued under a continuous time approach is represented for comparison (assuming a sampling time of 0.01 second). It is observed that in the first seconds, behaviors for both the event-triggered and continuous time approach are close. However, from the second 7, a difference can be observed that denotes the reduction in the transmission times. Considering that there is a sampling time of 0.01 second and a total simulation time of 100 seconds, then under the continuous time approach, there would be 10000 transmissions from each of the agents to their neighbors. With the event-triggered approach, it was obtained that agent number 1 transmitted 1012 times; agent 2 transferred 1095 times, agent 3 sent 1233 times and agent 4 transmitted 1328 times. Furthermore, an increase in the rate of transmission between agents takes place due to the introduced disturbances. With the support of figure 7 , it is clear that there exists a considerable reduction in the communication between agents, even in the presence of disturbances, in comparison with a continuous approach.

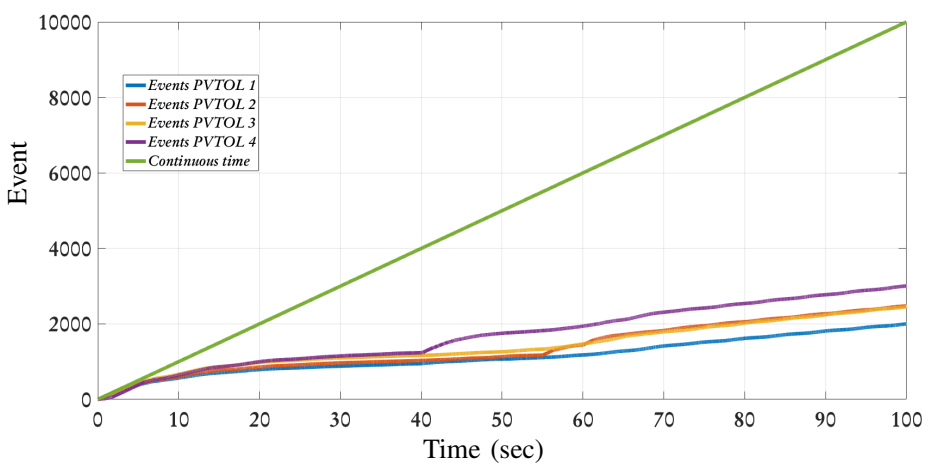

Fig. 7. The number of events

Figure 8 shows the evolution of the agents' positions on the plane $(x, y)$. The movements of each one of the agents are observed from their initial conditions until they reach the desired formation introduced in Table I.

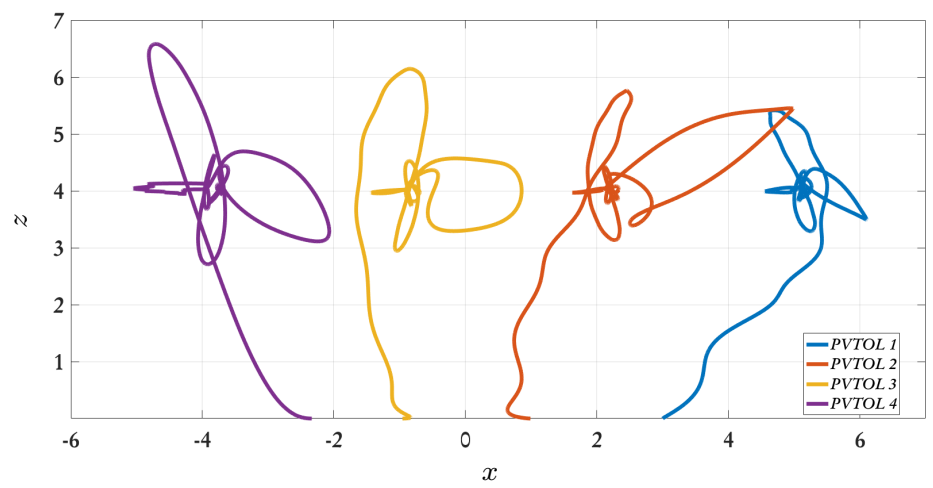

Fig. 8. Center of mass evolution for each UAV in the plane

\section{CONCLUSION}

In this work, an event-triggered cooperative control strategy applied to the problem of consensus and formation of a group of PVTOL carrying a suspended load was proposed. The control strategy was tested on simulation. The control strategy is two-layer based, and although inner layer is responsible of attitude stability which is an essential point of this work, we firmly believe that the most significant contribution is the development of an event-triggered control strategy used in the transmission of states between agents. The communication topology is represented by directed and connected graphs which represent realistic scenarios. This event-triggered control strategy reduces the number of transfers between agents, which demonstrate the superiority in terms of load on the communication medium. The control law, together with the event-trigger function, is designed to ensure a Minimal inter-Sampling Interval (MSI)[18] and then to avoid zero inter-sampling time leading to Zeno phenomena at finite time. This property and real-time experiments will be addressed in future work.

\section{REFERENCES}

[1] R. Olfati-Saber and R. M. Murray, "Consensus problems in networks of agents with switching topology and time-delays," IEEE Transactions on Automatic Control, vol. 49, no. 9, pp. 1520-1533, Sep. 2004.

[2] W. Ren and R. W. Beard, Distributed consensus in multi-vehicle cooperative control. Springer, 2008.

[3] F. L. Lewis, H. Zhang, K. Hengster-Movric, and A. Das, Cooperative control of multi-agent systems: optimal and adaptive design approaches. Springer Science \& Business Media, 2013.

[4] N. Ahmed, J. Cortes, and S. Martinez, "Distributed control and estimation of robotic vehicle networks: Overview of the special issue," IEEE Control Systems, vol. 36, no. 2, pp. 36-40, 2016.

[5] — "Distributed control and estimation of robotic vehicle networks: Overview of the special issue-part II," IEEE Control Systems, vol. 36, no. 4, pp. 18-21, 2016.

[6] J. Thunberg, J. Goncalves, and X. Hu, "Consensus and formation control on se(3) for switching topologies," Automatica, vol. 66, pp. 109 - 121, 2016. [Online]. Available: http://www.sciencedirect.com/ science/article/pii/S0005109815005671

[7] X. Peng, K. Guo, and Z. Geng, "Full state tracking and formation control for under-actuated vtol uavs," IEEE Access, vol. 7, pp. 3755-3766, 2019.

[8] H. Du, W. Zhu, G. Wen, Z. Duan, and J. L, "Distributed formation control of multiple quadrotor aircraft based on nonsmooth consensus algorithms," IEEE Transactions on Cybernetics, vol. 49, no. 1, pp. 342-353, Jan 2019. 
[9] M. M. Nicotra, E. Garone, R. Naldi, and L. Marconi, "Nested saturation control of an uav carrying a suspended load," in 2014 American Control Conference, June 2014, pp. 3585-3590.

[10] C. Meissen, K. Klausen, M. Arcak, T. I. Fossen, and A. Packard, "Passivity-based formation control for uavs with a suspended load," IFAC-PapersOnLine, vol. 50, no. 1, pp. 13150 - 13 155, 2017, 20th IFAC World Congress.

[11] A. Pulido-Flores, J. F. Guerrero-Castellanos, J. Linares-Flores, S. E. Maya-Rueda, J. U. Alvarez-Muoz, J. Escareno, and G. Mino-Aguilar, "Active disturbance rejection control for attitude stabilization of multi-rotors uavs with bounded inputs," in 2018 International Conference on Unmanned Aircraft Systems (ICUAS), 2018.

[12] S. Monaco and D. Normand-Cyrot, "Advanced tools for nonlinear sampled-data systems' analysis and control," in Control Conference (ECC), 2007 European, July 2007, pp. 1155-1158.

[13] K.-E. Årzén, "A simple event-based PID controller," in Preprints 14th World Congress of IFAC, Beijing, P.R. China, Jan. 1999.

[14] K. J. Åström and B. Bernhardsson, "Comparison of riemann and lebesque sampling for first order stochastic systems," in Proceedings of the 41st IEEE Conference on Decision and Control, 2002, vol. 2. IEEE, 2002, pp. 2011-2016.

[15] P. Tabuada, "Event-triggered real-time scheduling of stabilizing control tasks," Automatic Control, IEEE Transactions on, vol. 52, no. 9, pp. 1680-1685, 2007.

[16] A. Anta and P. Tabuada, "To sample or not to sample: Self-triggered control for nonlinear systems," Automatic Control, IEEE Transactions on, vol. 55, no. 9, pp. 2030-2042, 2010.

[17] M. Mazo Jr, A. Anta, and P. Tabuada, "On self-triggered control for linear systems: Guarantees and complexity," in Control Conference (ECC), 2009 European. IEEE, 2009, pp. 3767-3772.

[18] N. Marchand, S. Durand, and J. F. Guerrero-Castellanos, "A general formula for event-based stabilization of nonlinear systems," Automatic Control, IEEE Transactions on, vol. 58, no. 5, pp. 1332-1337, 2013.

[19] D. V. Dimarogonas, E. Frazzoli, and K. H. Johansson, "Distributed event-triggered control for multi-agent systems," IEEE Transactions on Automatic Control, vol. 57, no. 5, pp. 1291-1297, 2012.

[20] G. S. Seyboth, D. V. Dimarogonas, and K. H. Johansson, "Event-based broadcasting for multi-agent average consensus," Automatica, vol. 49 , no. 1, pp. 245-252, 2013.

[21] E. Garcia, Y. Cao, and D. W. Casbeer, "Decentralized event-triggered consensus with general linear dynamics," Automatica, vol. 50, no. 10 pp. $2633-2640,2014$.

[22] C. Nowzari and J. Cortes, "Team-triggered coordination for real-time control of networked cyber-physical systems," IEEE Transactions on Automatic Control, vol. 61, no. 1, pp. 34-47, Jan 2016.

[23] M. Zimmerling, L. Mottola, P. Kumar, F. Ferrari, and L. Thiele, "Adaptive real-time communication for wireless cyber-physical systems," ACM Trans. Cyber-Phys. Syst., vol. 1, no. 2, pp. 8:1-8:29, Feb. 2017.

[24] M. Muehlebach and S. Trimpe, "Distributed event-based state estimation for networked systems: An LMI approach," IEEE Transactions on Automatic Control, vol. 63, no. 1, pp. 269-276, Jan 2018.

[25] C. Nowzari, E. Garcia, and J. Cortes, "Event-triggered communication and control of networked systems for multi-agent consensus," Automatica, vol. 105, pp. 1 - 27, 2019. [Online]. Available: http://www.sciencedirect.com/science/article/pii/S000510981930130X

[26] J. Guerrero-Castellanos, J. Téllez-Guzmán, S. Durand, N. Marchand, J. Alvarez-Muñoz, and V. R. Gonzalez-Diaz, "Attitude stabilization of a quadrotor by means of event-triggered nonlinear control," Journal of Intelligent \& Robotic Systems, vol. 73, no. 1-4, pp. 123-135, 2014.

[27] J. F. Guerrero-Castellanos, A. Vega-Alonzo, N. Marchand, S. Durand, J. Linares-Flores, and G. Mino-Aguilar, "Real-time event-based formation control of a group of vtol-uavs," in 2017 3rd International Conference on Event-Based Control, Communication and Signal Processing (EBCCSP), May 2017, pp. 1-8.

[28] R. Olfati-Saber and J. S. Shamma, "Consensus filters for sensor networks and distributed sensor fusion," in Decision and Control, 2005 and 2005 European Control Conference. CDC-ECC'05. 44th IEEE Conference on. IEEE, 2005, pp. 6698-6703.
[29] J. F. Guerrero-Castellanos, N. Marchand, A. Hably, S. Lesecq, and J. Delamare, "Bounded attitude control of rigid bodies: Real-time experimentation to a quadrotor mini-helicopter," Control Engineering Practice, vol. 19, no. 8, pp. 790-797, 2011.

[30] A. R. Teel, "Global stabilization and restricted tracking for multiple integrators with bounded controls," Systems and Control Letters, pp. 165-171, 1992. 